\title{
Persistent Hypotony Post Vitrectomies - A Case Report
}

\author{
Aina Malindri Dasrilsyah ${ }^{1}$, Ainal Adlin Naffi ${ }^{2}$, Mae-Lynn Catherine Bastion ${ }^{3}$ \\ 1,2,3 Department of Ophthalmology, Universiti Kebangsaan Malaysia Medical Centre \\ (UKMMC), Kuala Lumpur, Malaysia.
}

\section{INTRODUCTION}

Hypotony is defined as low intraocular pressure (IOP) which leads to functional and structural changes of the eye, mainly optic nerve, choroid and retina in the posterior pole. There are two types of hypotony which are clinical and statistical hypotony. "Statistical hypotony" refers to IOP which is less than $6.5 \mathrm{mmHg}$, more than 3 standard deviations below the mean IOP, ${ }^{1}$ whereas, "Clinical hypotony" refers to IOP that is low enough to result in loss of vision and it can be caused by structural changes such as astigmatism, corneal oedema, cystoid macular oedema or maculopathy. ${ }^{2}$ Hypotony maculopathy is characterised by chorioretinal folds, acute optic nerve head oedema and tortuous retinal veins. ${ }^{3}$

The aetiologies can be due to either increased outflow of aqueous humour, for example in surgical wound leak, over filtrating bleb and cyclodialysis cleft or reduced aqueous humour production, for example in inflammatory conditions. 4,5

The risk factors are male gender, young age, myopia, primary glaucoma filtering surgery, especially with the usage of antifibrotic agents, multiple ocular surgeries, vitrectomy and elevated preoperative intraocular pressure.6,7,8

In the present report, we describe a case of a middle-aged gentleman with a background of high myopia who underwent multiple ocular surgeries and presented with postoperative persistent clinical hypotony.
Corresponding Author: Dr. Ainal Adlin Naffi, Department of Ophthalmology, Universiti Kebangsaan Malaysia Medical Centre (UKMMC),

Jalan Yaacob Latif - 56000 Cheras, Kuala Lumpur, Malaysia. E-mail: ainaladlin@yahoo.com

DOI: $10.14260 /$ jemds/2022/55

How to Cite This Article: Dasrilsyah AM, Naffi AA, Bastion MLC. Persistent hypotony post vitrectomies - a case report. J Evolution Med Dent Sci 2022;11(01):298-301, 10.14260/jemds/2022/55

Submission 02-12-2021,

Peer Review 05-01-2022,

Acceptance 14-01-2022,

Published 31-01-2022.

Copyright (C) 2022 Aina Malindri Dasrilsyah et al. This is an open access article distributed under Creative Commons Attribution License [Attribution 4.0 International (CC BY 4.0)] 


\section{PRESENTATION OF CASE}

A 59-year-old gentleman with high myopia (-10.0 Dioptre) had his right eye cataract surgery done eight years and left eye surgery done seventeen years ago. Both surgeries were uncomplicated. He gained good vision postoperatively with the best-corrected visual acuity (BCVA) of $6 / 6$ bilaterally.

$\mathrm{He}$ was well until he developed pseudophakic rhegmatogenous retinal detachment of the right eye, nine months following the cataract surgery and underwent right eye combined scleral buckle, trans pars plana vitrectomy (TPPV) with $\mathrm{C}_{3} \mathrm{~F}_{8}$ gas tamponade. He presented again with right eye inferior retinal re-detachment with the macula off six weeks after the first vitrectomy. He then underwent a right eye vitrectomy with retinectomy and silicone oil tamponade. Postoperatively, the BCVA of the right eye was $6 / 24$. The silicone oil was removed three months later. His IOP trend during the post-operative period ranged between 4-12 mmHg.

His right eye was stable until he presented again with a subluxated intraocular lens seven years after removal of the silicone oil. The intraocular lens was explanted, and the scleral fixated intraocular lens was implanted following that.

Since day one post-surgery, his intraocular pressure was low, ranging 2-7 $\mathrm{mmHg}$ without any other significant findings. His intraocular pressure remained low until two weeks post-surgery with $6 \mathrm{mmHg}$. His visual acuity improved from counting fingers (CF) to $3 / 60$ (pinhole 6/24). There was no corneal oedema. The optic disc was oedematous, however, there was no choroidal effusion, chorioretinal folds or vascular tortuosity seen.

Gonioscopy did not show a cyclodialysis cleft. Anterior segment optical coherence tomography (AS-OCT) showed no ciliary body detachment (Figure 1). Bjerrum showed an incongruous right upper quadrantanopia field defect (Figure 2), Farnsworth D15 test showed a normal result. Blood investigations including complete blood count (CBC), erythrocyte sedimentation rate (ESR), fasting blood sugar (FBS), fasting serum lipid (FSL), chest x-ray, urine full examination microscopy examination (UFEME) were taken to rule out other causes of optic disc swelling but they were unremarkable.

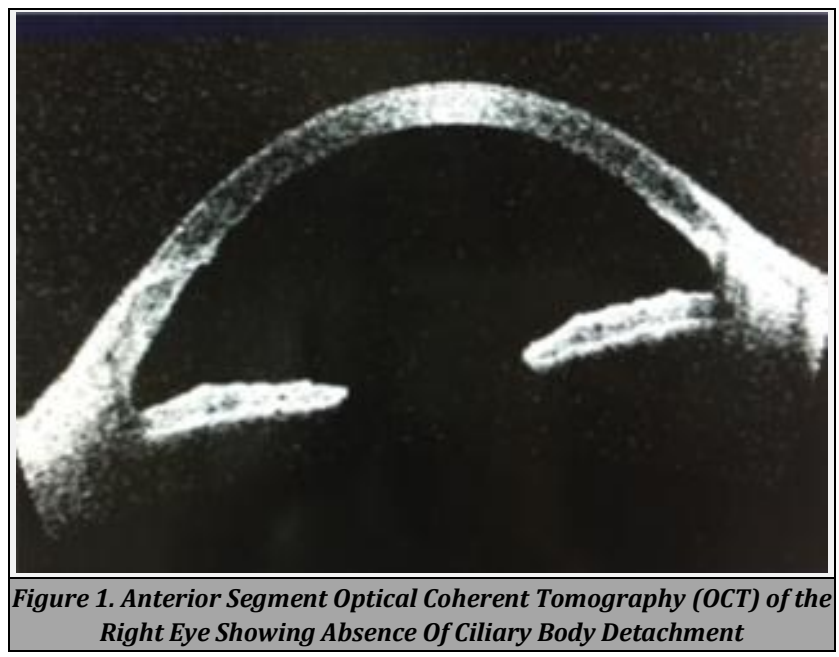

On week three post-operatively, we noticed that his IOP remained low. Hence, we started him on a slow tapering dose of oral prednisolone $30 \mathrm{mg}$ OD and he completed six weeks of treatment. He showed visual recovery after six weeks of treatment with BCVA of 6/12. Intraocular pressure (IOP) remained low at $5 \mathrm{mmHg}$ and the optic disc showed residual oedema temporally. He was followed up until six months postoperatively and his latest IOP was $14 \mathrm{mmHg}$ with no more residual oedema of the optic disc.

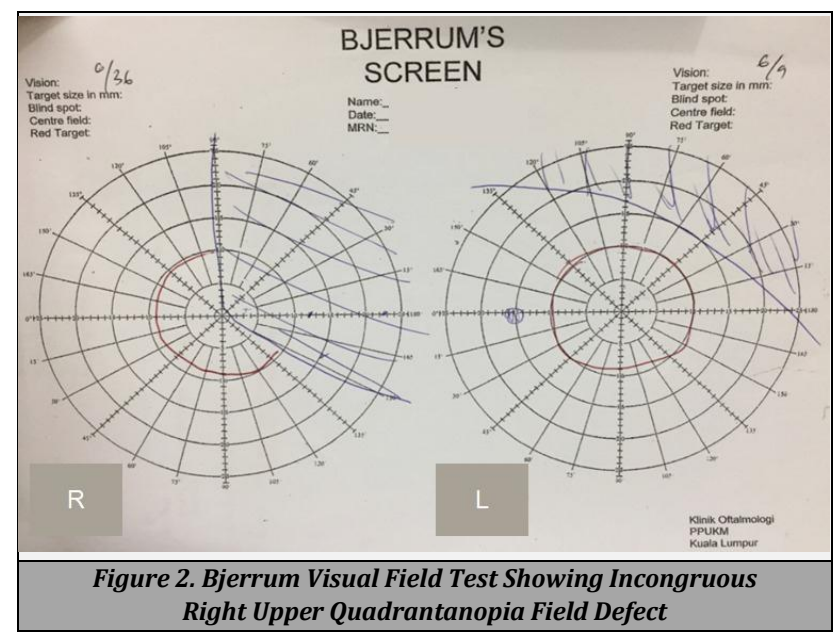

DISCUSSION

This report describes a case of persistent hypotony in high myopia patient with a previous history of multiple ocular surgeries. With no other risk factors such as glaucoma filtrating surgery, usage of antifibrotic agents or preoperative high intraocular pressure (IOP), this points towards vitrectomy related clinical hypotony.

Mimouni et al. reported a higher number of previous vitreoretinal surgeries in the hypotony group (1.5 vs. $0.4, \mathrm{P}<$ 0.001). ${ }^{8}$ Giulio et al. described the incidence of hypotony more often in reoperations (29.9\%) compared to primary operations (9.2\%; statistically different). ${ }^{9}$ This is similar to our patient who underwent three vitrectomy surgeries on his right eye over seven years.

Patients with clinical hypotony typically present with reduced visual acuity. Loss of central vision and distortions also occur if the macula is involved. On clinical examinations, there might be the presence of optic nerve oedema associated with chorioretinal folds in the posterior pole. Apart from clinical findings, optical coherence tomography (OCT) of macula also plays an important role in diagnosing hypotony maculopathy. OCT macula is helpful in those patients with reduced visual acuity but the absence of fundus findings. Budenz et al. described three cases of hypotony maculopathy that showed normal ocular examination but reduced in their best-corrected visual acuity, and all these cases revealed retinal and/or choroidal folding in the OCT. ${ }^{10}$

The maculopathy from the OCT resolved after normalization of the IOP. Kokame et al. also reported cases of resolved macular oedema on OCT when the IOP got normalized. ${ }^{9}$ Our patient presented with reduced visual acuity without loss of central vision and distortions. Examinations showed optic nerve oedema, but there were no chorioretinal folds in the posterior pole seen clinically and 
from OCT. He also had improved visual acuity when IOP normalized.

Another risk factor identified for postoperative hypotony is the removal of the silicone oil from a silicone oil-filled eye. ${ }^{11}$ The ciliary body has been accustomed to a lower rate of production in these eyes due to the presence of silicone oil globule that occupies most of the vitreous cavity. When the oil is subsequently removed, the ciliary body may take a few days up to one week before the rate of aqueous production is increased to match that needed for the posterior segment. Aqueous production may also be low in response to ocular inflammation that may be excessive in eyes with multiple surgeries or complex retinal detachments; a form of ciliary shut-down. Another cause could be due to increased aqueous outflow which is associated with increased levels of prostaglandins in ocular inflammation. However, in our patient, the silicone oil removal was seven years before as well as the instrumentation used for the removal of the dropped lens and the implantation of the scleral tunnel fixed IOL was not overly excessive.

Fundus fluorescein angiography (FFA) also can demonstrate the presence of chorioretinal folds and differentiate the choroidal folds from the retinal folds. The features of FFA are an irregular increase in background choroidal fluorescence which produces a series of hyperfluorescent streaks corresponding to the crests of the choroidal folds and the troughs of the folds appear hypofluorescent. There is also some leakage from the optic nerve capillaries but usually not from the retinal capillaries. ${ }^{4}$

However, in our patient, due to the absence of choroidal fold clinically, and as the patient responded well with oral prednisolone, FFA was not done.

Ultrasound biomicroscopy (UBM) is also an important tool in further evaluation of ocular hypotony. It is used to assess iris contour, anterior chamber depth, as well as visualization of anatomical structures posterior to the iris. Useful information can be obtained based on the position of the ciliary body and/or its anterior relation. Besides that, UBM may also detect cyclodialysis cleft when missed clinically. Marcio et al. reported almost all chronic hypotony cases that have undergone pars plana vitrectomy (PPV) presented with ciliary body alterations. However, this test was not done on our patient.

Vitrectomy is also one of the risk factors for hypotony and the rate is about $5.2-13.1 \%$ at day 1 post-operatively. ${ }^{7,8}$ In addition, Giulio et al. described that usage of intravitreal triamcinolone increased the incidence of hypotony post vitrectomy compared to cases without triamcinolone (35.3\% versus $10.3 \%$ ). This result was an unexpected finding from the study as usage of corticosteroids was expected to cause an increase in intraocular pressure. They also reported that phakic eyes had significantly less chance of hypotony (6.7 \%) compared to pseudophakic eyes (15.6\%) and eyes undergoing combined phacoemulsification and vitrectomy $(25.0 \%) .^{12}$ In our patient, he had a previous history of three vitrectomies as well as the pseudophakic eye.

Due to reoperations, he was more susceptible to hypotony. Shimada et al. ${ }^{13}$ postulated that more fluid leakage happens in reoperations compared to primary operations. The previous surgery could alter the regenerative capacity and elasticity of scleral tissue, causing a wound in the tissue more susceptible to leakage. In addition, less internal vitreous plugging of sclerotomies was achieved in revitrectomised eyes due to more complete excision of the vitreous.

Furthermore, in our patient, other risk factors identified that contributed to hypotony maculopathy were male gender, myopia and young age (less than 60 years of age) as similar to a study conducted by Fannin et al. ${ }^{14}$

Reduced scleral rigidity in these groups of patients is one of the postulations which contribute to the collapse of the scleral wall during hypotony, causing chorioretinal folds. ${ }^{4}$

A lower risk of hypotony occured with the usage of tamponade compared to no tamponade during vitrectomy. The risk of hypotony in cases with gas or air tamponade was $3.3 \%$ than in cases without tamponade which was $22.4 \%$ in post vitrectomy cases. ${ }^{9}$

In the Silicone Study, ${ }^{15}$ hypotony was reported in $31 \%$ of patients treated with gas, and only in $18 \%$ treated with silicone oil. Reda Issa et al. observed hypotony in $8 \%$ of eyes after silicone oil removal. Mimouni et al. reported that silicone oil removal increased the risk for early postoperative hypotony after vitrectomy (61.1\% vs. $8.3 \%, \mathrm{P}<0.001) .{ }^{8}$ In our patient, the short duration of silicone oil tamponade of three months could be the possible cause of hypotony.

Another interesting postulation for this patient is the possibility that the patient's scleral buckles resulted in subclinical anterior segment ischemia which was predisposed to zonulolysis. This same ischemia could have caused ciliary body ischemia; hence the low aqueous production level and low IOP were encountered in this case.

However, other signs of anterior segment ischemia such as iris atrophy, rubeosis and persistent anterior segment inflammation were not seen. Intraocular lens dislocation is otherwise commonly encountered in high myopia and vitrectomy surgery. ${ }^{16}$ The postulation behind high myopia causing intraocular lens dislocation is the longer axial length of the eyeball causing 360-degree zonular fibres stretching and dehiscence, that fails to adequately resist the tension of the anterior capsule contraction or the zonular dialysis. Vitrectomy surgery for retinal detachment is more likely to cause intraocular lens dislocation than vitrectomy for macular disease. The postulation could be due to aggressive peripheral vitrectomy with scleral depression during the vitrectomy for retinal detachment that may damage the posterior zonular fibres.

For the management of hypotony maculopathy, it is crucial to correctly identify the cause of hypotony and treat the cause as soon as possible. Delayed treatment can cause permanent changes in the retina and choroid, which subsequently leads to poor visual prognosis. ${ }^{17}$

\section{CONCLUSIONS}

In conclusion, multiple ocular surgeries are known risk factors for persistent hypotony. Although it was a highly suspected cause in our patient, thorough investigations still need to be done for further management to prevent loss of vision.

Financial or other competing interests: None.

Disclosure forms provided by the authors are available with the full text of this article at jemds.com. 


\section{REFERENCES}

[1] Pederson JE. Ocular hypotony. In: Ritch R, Krupin T, Shields MB, eds. The glaucomas. $2^{\text {nd }}$ edn. Mosby: St. Louis 1996:385-95.

[2] Schubert HD. Postsurgical hypotony: relationship to fistulization, inflammation, chorioretinal lesions, and the vitreous. Surv Ophthalmol 1996;41(2):97-125.

[3] American Academy of Ophthalmology. Hypotony maculopathy.

https://eyewiki.aao.org/Hypotony_Maculopathy

[4] Gass JD. Hypotony maculopathy. In: Bellows JG, ed. Contemporary ophthalmology. Honoring Sir Stewart Duke-Elder. Baltimore: Williams \& Wilkins 1972:343-66.

[5] Minckler DS, Bunt AH. Axoplasmic transport in ocular hypotony and papilloedema in the monkey. Arch Ophthalmol 1997;95(8):1430-6.

[6] Fannin LA, Schiffman JC, Budenz DL. Risk factors for hypotony maculopathy. Ophthalmology 2003;110(6):1185-91.

[7] Bamonte G, Mura M, Tan HS. Hypotony after 25-gauge vitrectomy. Am J Ophthalmol 2011;151(1):156-60.

[8] Mimouni M, Abualhasan H, Derman L, et al. Incidence and risk factors for hypotony after 25-gauge pars plana vitrectomy with nonexpansile endotamponade. Retina 2018;40(1):41-6.
[9] Kokame GT, de Leon MD, Tanji T. Serous retinal detachment and cystoid macular edema in hypotony maculopathy. Am J Ophthalmol 2001;131(3):384-6.

[10] Budenz DL, Schwartz K, Gedde SJ. Occult hypotony maculopathy diagnosed with optical coherence tomography. Arch Ophthalmol 2005;123(1):113-4.

[11] Kim SW, Oh J, Yang KS, et al. Risk factors for the development of transient hypotony after silicone oil removal. Retina 2010;30(8):1228-36.

[12] Bamonte G, Mura M, Tan HS. Hypotony after 25-gauge vitrectomy. Am J Ophthalmol 2011;151(1):156-60.

[13] Shimada H, Nakashizuka H, Mori R, et al. 25-gauge scleral tunnel transconjunctival vitrectomy. Am J Ophthalmol 2006;142(5):871-3.

[14] Fannin LA, Schiffman JC, Budenz DL. Risk factors for hypotony maculopathy. Ophthalmology 2003;110(6):1185-91.

[15] Barr CC, Lai MY, Lean JS, et al. Postoperative intraocular pressure abnormalities in the Silicone Study. Silicone Study Report 4. Ophthalmology 1993;100(11):1629-35.

[16] Fan Q, Han X, Zhu X, et al. Clinical Characteristics of intraocular lens dislocation in Chinese Han populations. Journal of Ophthalmology 2020;2020:8053941.

[17] Nuyts RM, Greve EL, Geijssen HC, et al. Treatment of hypotonous maculopathy after trabeculectomy with mitomycin C. Am J Ophthalmol 1994;118(3):322-31. 